\title{
Fair Power-Controlled Multiple Access in Mobile Ad Hoc Networks
}

\author{
Wenjian Shao, Dongxu Shen, Daiqin Yang and Victor O. K. Li \\ Department of Electrical and Electronic Engineering \\ The University of Hong Kong \\ Pokfulam, Hong Kong, China \\ \{wjshao, dxshen, dqyang, vli\}@eee.hku.hk
}

\begin{abstract}
Previous power-controlled media access control (MAC) protocols for mobile ad hoc networks (MANET) suffer from the unfair channel access problem, i.e., it is difficult for a node to communicate with a distant node because of nearby ongoing communications. In this paper, we propose a fair power-controlled MAC protocol (FPCMA) by integrating senderinitiated busy tone with traditional power-controlled protocol. The sender-initiated busy tone is used to assist in channel access, and will be set up when a node finds it difficult to access the channel. Nearby nodes overhearing the tone will yield their transmission rights to the attempting node, thus solving the unfairness problem. Through analysis and simulation, we demonstrate that our protocol is able to balance the tradeoff between fair channel access and throughput, and is therefore more flexible than both the traditional dual busy tone and power-controlled MAC protocols.
\end{abstract}

\section{INTRODUCTION}

A central issue in improving the efficiency of mobile ad hoc network (MANET) is to design effective media access control (MAC) protocols. An ideal design of MANET MAC should achieve high throughput, and allow fair channel access for all users. The two most important issues that can significantly reduce channel utilization are the well-known hidden terminal and exposed terminal problems. These two problems are essentially caused by the limited awareness of transmission/reception situation around each mobile terminal, which is inevitable due to the inherent distributed nature of MANET.

Much MAC layer research revolves around the solution of the above two issues, with the goal of improving channel utilization. MANET MAC protocols can be generally divided into power-controlled and non-power-controlled categories. Nonpower-controlled protocols are represented by IEEE 802.11 MAC [1], split-channel protocols [2][3], busy tone based protocols [4][5], etc. Power controlled schemes include [6][7], etc. However, existing work either provide a partial solution to the hidden/exposed terminal problem [1][2][3][4], or spawn new problems such as unfairness in channel access [6][7].

Although IEEE 802.11 is the de facto standard for the study of ad hoc networks, it does not work well in MANET since it is originally designed for wireless LAN. It suffers from the well-known hidden terminal and exposed terminal problems in ad hoc networks, thus leading to low channel utilization. To improve the performance of IEEE 802.11, the split channel schemes [2][3] introduce a separate control channel besides the data channel. The throughput of the whole system heavily depends on the traffic model and the bandwidth allocation method between the two channels. Furthermore, these protocols still suffer from the hidden/exposed terminal problems.

The busy tone protocols [4][5] use busy tone to advertise the status of each node, thus effectively solving the hidden/exposed terminal problems. A major drawback of the busy tone protocols is that the transmission power is always fixed, regardless of communication distance. Thus bandwidth is not efficiently reused.

The idea of power controlled schemes [6][7] is to adjust the transmission power optimally so that interference can be reduced as much as possible. With proper transmission power calculation, channels can be aggressively reused, thus significantly boosting throughput. The biggest problem with PCMA (power controlled multiple access)[6] and its variants [7] is the unfairness problem under heavy traffic load. If a node wants to communicate with a far-away node, a high power should be used. Unfortunately, in PCMA, a high-power node is always at a disadvantage to grab the channel, and this channel access probability will monotonically decrease with the increase of the traffic load. Therefore, when the traffic load is extremely high, distant nodes may be unable to communicate with each other, which will eventually result in a partitioned network.

In this paper, we propose a fair power controlled multiple access protocol (FPCMA). We tackle the unfairness problem by utilizing a sender-initiated power-controlled busy tone as an additional declaration mechanism for blocked nodes. When a node finds it difficult to reach a distant node, it turns on the sender-initiated busy tone using a properly selected power. Overhearing the busy tone, nearby nodes will yield their transmission rights to the blocked node. Another problem with PCMA is that the request-to-send (RTS) control packets are unprotected and the clear-to-send (CTS) control packets are likely to be blocked. Therefore, we also adopt the dual busy tone technique in DBTMA [5] to reduce the collision of RTS packets and eliminate the CTS blocking problem. Simulation results show that our approach can achieve good fairness with acceptable reduction of throughput. By properly selecting protocol parameters, we can trade off throughput for fairness. Therefore, our protocol achieves a balance between DBTMA and PCMA.

This paper is organized as follows. In Section II, we discuss the related work. In Section III, we present our MAC protocol design. The fairness problem and our solution are presented 
in Section IV. Simulation results are shown in Section V and Section VI is the conclusion.

\section{RELATED WORK}

\section{A. 802.11 Based Protocols}

The 802.11 MAC uses both virtual and physical carrier sensing to treat the hidden terminal problem. Virtual carrier sensing is carried out through RTS/CTS control packets which indicate the transmission duration. Neighboring nodes will keep silent during the announced transmission period, under the condition that RTS/CTS packets are correctly decoded. However, terminals that can receive but are unable to correctly decode the RTS/CTS packets will be unaware of the actual transmission time, and thus become hidden terminals. According to [5], CTS further suffers from collisions, and as high as $60 \%$ of CTS packets will be destroyed under heavy traffic load. Due to the above reasons, the hidden terminal problem still exists in 802.11. Moreover, 802.11 has no mechanism to deal with the exposed terminal problem.

Many protocols have been proposed to improve the performance of 802.11. One approach is the split channel scheme [2][3], in which a single channel is split into two sub-channels: a data sub-channel and a control sub-channel. Control packets, RTS/CTS/ACK, are sent on the control sub-channel, while data packets are transmitted over the data sub-channel. By moving the contention resolution phase to a narrow control sub-channel, the data sub-channel transmission is free of contention. However, the throughput of the whole system heavily depends on the traffic model and the bandwidth allocation algorithm between the two channels. Even with a dedicated control sub-channel, the above hidden terminal problem still remains.

\section{B. Busy Tone Based Protocols}

In [4], Wu and Li propose a receiver-initiated busy-tone multiple access protocol (RI-BTMA). In this protocol, after receiving a preamble from the transmitter (the preamble is similar to the role of RTS), the receiver sets up an out-of-band busy tone, which will last until the end of DATA reception. The busy tone serves two functions. First, it is an acknowledgement to the preamble, similar to CTS. The second and the most important function is to eliminate the hidden terminals, i.e., whoever detects the busy tone will back off, without the need of decoding. In this way, hidden terminals are eliminated.

DBTMA [5] uses one transmit and one receive busy tone $\left(B T_{t}\right.$ and $\left.B T_{r}\right)$ to solve the hidden and exposed terminal problems. When a node, say node $\mathrm{A}$, has packets to send to node $\mathrm{B}$, it first senses $B T_{t}$ and $B T_{r}$. If either busy tone exists, it will back off its transmission. Otherwise, node A turns on $B T_{t}$ and transmits an RTS packet. When node $\mathrm{B}$ receives RTS, it turns on $B T_{r}$ as a reply indication. When node A senses $B T_{r}$, it turns off $B T_{t}$ and transmits a DATA packet. Since $B T_{r}$ is turned on during the reception, other nodes that sense it will back off to avoid collision, thus resolving the hidden terminal problem. On the other hand, the exposed terminal problem is solved since a node which does not sense $B T_{t}$ and $B T_{r}$ can still initiate its transmission even though the channel is busy.

\section{Power Controlled MAC Protocols}

In [6], Monks et al. propose PCMA. In PCMA, each node needs to calculate the maximum allowable power for its transmission without interfering with other ongoing receptions. This is called power bounding. The power bound $\left(P t_{\text {bound }}\right)$ is designed such that after propagation loss, the received power will not exceed the tolerable additional noise $\left(N_{a}\right)$ of all neighboring receivers. $N_{a}$ is decided by

$$
N_{a}=\max \left\{\frac{P r_{R T S}}{S I R \_ \text {Thresh }}-P_{n}, \text { CS_Thresh }\right\}
$$

where $\operatorname{Pr}_{R T S}$ is the received power of RTS, SIR_Thresh is the signal-to-interference ratio threshold for correct packet reception under interference, CS_Thresh is the carrier sensing threshold for clear channel assessment, and $P_{n}$ is the noise power at the receiver. To announce its $N_{a}$, each receiver adopts a receiver-initiated power-controlled busy tone $\left(B T_{r}\right)$, whose transmission power $\left(P t_{B T r}\right)$ is given by

$$
P t_{B T r}=\frac{P t_{\max } \cdot C S_{\_} \text {Thresh }}{N_{a}}
$$

where $P t_{\max }$ is the maximal transmission power. From (2), we can see $P t_{B T r}$ is inversely proportional to $N_{a}$. According to the maximal received power on $B T_{r}$ channel $\left(P r_{B T r}\right)$, each node can identify the most vulnerable receiver around it and decide its $P t_{\text {bound }}$ by

$$
P t_{\text {bound }}=\min \left\{\frac{P t_{\max } \cdot C S_{-} \text {Thresh }}{\max \left\{P r_{B T r}\right\}}, P t_{\max }\right\}
$$

In PCMA, a transmitter sends a request using its $P t_{\text {bound }}$. After receiving the request, the receiver calculates the desired power $\left(P t_{d e s}\right)$ and $N_{a}$ for the transmission according to the channel gain and local noise level. If $P t_{\text {bound }} \geq P t_{\text {des }}$, the receiver accepts the request and data are sent with $P t_{d e s}$.

PCMA can achieve higher throughput than DBTMA, especially at high load. In PCMA, nodes sensing the $B T_{r}$ are not simply forbidden to access the channel as in DBTMA. Instead, they access the channel as long as the transmission power is lower than $P t_{\text {bound }}$. However, a serious problem in PCMA and other power-controlled MAC protocols is the unfairness problem under heavy traffic load. When a node is to communicate with a distant node, its $P t_{d e s}$ is more likely to exceed its $P t_{\text {bound }}$. This is especially true when the load is heavy, i.e., there are many nearby concurrent transmissions, $P t_{\text {bound }}$ may be constrained to a low level and consequently distant pairs of nodes may be unable to establish a link. Therefore, links with short distance are easy to set up, and it is unfair for nodes over long distance.

In PCMA, there is no protection for RTS/CTS packets, which will suffer from collisions. Another problem for PCMA is CTS blocking. When a receiver is too close to another receiver, it will be blocked from replying CTS due to power bounding.

\section{FPCMA: BASIC MODE}

\section{A. Design Motivations}

From the literature review, we conclude that PCMA is most effective in achieving high channel throughput. However, it still has a number of drawbacks. 
Firstly, a common problem in power-controlled protocols, including [6] and [7], is the unfair channel access problem, which is very undesirable for large networks with high load. We analyze this problem in Section IV and introduce the fairness mode of our protocol, which uses the sender-initiated powercontrolled busy tone $\left(B T_{t}\right)$ as a mechanism for blocked nodes to announce the access request. With the help of $B T_{t}$, the unfairness problem can be solved.

Secondly, there is no protection for RTS/CTS packets, which will suffer from collisions. PCMA also suffers from the CTS blocking problem. When a receiver is too close to another receiver, it will be blocked from replying CTS due to power bounding. On the other hand, although the throughput of DBTMA is inferior to PCMA, the use of a $B T_{t}$ to protect RTS and substituting CTS by $B T_{r}$ are two merits. Thus, we can borrow these techniques to fix the problems in PCMA. In this section, we provide the basic mode of our protocol by incorporating dual busy tone with PCMA, which will further improve the throughput of PCMA.

\section{B. System Assumptions}

In this paper, we make the same assumptions on the channels as in PCMA [6]. They are:

- The channel gain remains unchanged during the transmission of RTS and DATA.

- The channel gain from node A to node B equals the channel gain from node $\mathrm{B}$ to node $\mathrm{A}$.

- The gain of the data channel equals the gain of the busy tone channel.

To avoid the overlapping of multiple tones, periodical busy tone pulses instead of a solid tone are used in the busy tone channel as in PCMA. According to [8], only 1-2\% of bandwidth is needed for busy tones to achieve the best performance of the system. Thus, we assume the overhead caused by busy tones is negligible. We also assume nodes know the required transmission power to the desired location (denoted as $P t_{d e s}$ ), which is decided by the receiver in PCMA. To determine $P t_{d e s}$, a sender must have the distance information between itself and the receiver. Many location-aware MAC protocols are proposed to help senders determine the distance to the desired destination, for example, through global positioning system (GPS). Distance also can be estimated through the previous transmissions, as discussed in [9].

\section{Basic Mode Description}

In DBTMA, when node B is to reply an RTS from node A, CTS is not transmitted since $B T_{r}$ is used as an indicator of acceptance. We borrow this idea in our protocol to solve the CTS blocking problem. However, because $B T_{r}$ signals contain no message, the problem is how node A can identify whether node $\mathrm{B}$ has replied when there are multiple $B T_{r}$ signals around. Our solution is to measure the variation of the received power on the $B T_{r}$ channel at node $\mathrm{A}$. If the received power on the $B T_{r}$ channel is increased by a factor of at least SIR_Thresh, node A can be convinced that node $\mathrm{B}$ has turned on its $B T_{r}$. A proof for this reply mechanism is provided in Appendix A.

The basic mode of our protocol works as follows.
1) When Node $\mathrm{A}$ is in idle state, it continuously monitors the $B T_{r}$ channel to update its $P t_{\text {bound }}$ according to (3).

2) When node $A$ has a data packet to send, it tries to sense the transmit busy tone $B T_{t}$. If $B T_{t}$ is present, which means other nodes are sending RTS, node A backs off to avoid collision; otherwise, node A checks whether its $P t_{\text {bound }} \geq P t_{\text {des }}$. If so, node A turns on its $B T_{t}$ using $P t_{\text {bound }}$ and sends RTS using $P t_{\text {bound }}$; otherwise, node A backs off.

3) When node $B$ receives the RTS packet, it calculates the additional noise it can tolerate and the transmission power for $B T_{r}$ according to (1) and (2). Then it turns on $B T_{r}$ using $P t_{B T r}$ as a reply indication.

4) Node A continuously monitors the $B T_{r}$ channel after transmitting RTS.

a) When it senses the received power on the $B T_{r}$ channel has increased by a factor of at least SIR_Thresh, it decides that node B has accepted the request.

b) Then node A turns off $B T_{t}$ and transmits the data packet using $P t_{d e s}$.

c) If the power on the $B T_{r}$ channel has not increased by $S I R \_T h r e s h$ for a certain period, node A will try to retransmit the packet starting from Step 1 .

5) During the reception of the data packet, node B continuously updates the transmission power of its $B T_{r}$, based on the measured interference.

6) Node B turns off $B T_{r}$ when it successfully receives the data packet.

\section{FPCMA: FAIRNESS MODE}

\section{A. Analysis of Unfairness Problem}

In this section, we study the probability that node A can successfully initiate a transmission to node $\mathrm{B}$ at distance $d$, $\operatorname{Pr}\{A \rightarrow B\}$. We can show that in PCMA, $\operatorname{Pr}\{A \rightarrow B\}$ is monotonically decreasing with the increase of $d$ and network traffic load $G$, which accounts for the unfairness problem.

To establish a link from A to B in PCMA, there has to be $P t_{\text {des }} \leq P t_{\text {bound }}$. Given $P t_{\text {bound }}, \operatorname{Pr}\{A \rightarrow B\}=$ $\operatorname{Pr}\left\{P t_{\text {des }} \leq P t_{\text {bound }}\right\}$. In an open space environment, the propagation model can be described as

$$
P_{r}=C_{k} \cdot P_{t} \cdot d^{-k}, \quad k \geq 2
$$

where $k \geq 2$ is the path loss exponent, $P_{t}$ is the transmission power, $P_{r}$ is the received power, and $d$ is the propagation distance, $C_{k}$ is a factor relating to physical and channel parameters. Obviously, $P t_{d e s} \propto d^{k}$, and $\operatorname{Pr}\{A \rightarrow B\}$ is monotonically decreasing with respect to $d^{k}$.

On the other hand, $P t_{\text {bound }}$ is related to the load $G$. As discussed previously, $P t_{\text {bound }}$ is determined by the most vulnerable receiver around A, say, node C. Pt bound is calculated according to a function that is inversely proportional to $\operatorname{Pr}_{B T r}(c)$, the received power of the busy tone pulse from $\mathrm{C}$, which is the strongest one on the $B T_{r}$ channel. Node $\mathrm{C}$ transmits busy tone pulses with power $P t_{B T r}(c), P t_{B T r}(c) \propto N_{a}^{-1}(c)$, where $N_{a}(c)$ is the additional noise node $\mathrm{C}$ can tolerate. Thus, 
$\operatorname{Pr}_{B T r}(c) \propto N_{a}^{-1}(c) \cdot l^{-k}(c)$, where $l(c)$ is the distance between A and C. Finally, we get $P t_{\text {bound }} \propto N_{a}(c) \cdot l^{k}(c)$. Obviously, both $N_{a}(c)$ and $l(c)$ are related to the traffic load $G$. When $G$ is high, $N_{a}(c)$ has to be small since interference is high. Similarly, $l(c)$ is also small because there is a high density of concurrent communications in the network. Therefore, $P t_{\text {bound }}$ and $\operatorname{Pr}\{A \rightarrow B\}$ are monotonically decreasing when $G$ gets high. From the above discussions, we can see that the access probability drops fast with the increase of $d$ and $G$. It is clear that short-range communications are more likely to survive in the contention because their $P t_{\text {des }}$ are much smaller than those of long-range communications. This problem is more serious when traffic load is high since $P t_{\text {bound }}$ is constrained to a small value.

\section{B. Fairness Mode Description}

The basic idea of our protocol is to use a sender-initiated busy tone $\left(B T_{t}\right)$ as a mechanism for blocked nodes to announce the access requests. A blocked node will set up $B T_{t}$ with power $P t_{d e s}$ to notify its neighbors that it wants to send packets using this power. Its neighbors sensing $B T_{t}$ will consider it as an urgent, high priority request, and backoff their transmissions.

The fairness mode steps are:

1) When node A fails to access the channel for more than a given threshold (represented as $F$ Thresh) number of times, it turns on $B T_{t}$ using $P t_{\text {des }}$ until it can access the channel.

2) If a node senses the $B T_{t}$ channel with maximal received power $\left(\operatorname{Pr}_{B T t}\right)$ larger than the threshold for correct packet decoding (RX_Thresh), it will both backoff its transmission and reject any RTS request until this periodical pulse disappears. Else, it will add the received pulse power to its noise level on the data channel to account for the interference from A when A transmits later.

3) After node A turns on its $B T_{t}$, it continuously senses the $B T_{r}$ channel and updates its $P t_{\text {bound }}$. When $P t_{\text {bound }} \geq$ $P t_{d e s}$, it turns off its $B T_{t}$ and tries to access the channel using the basic mode.

In our protocol, since nodes sensing $P r_{B T t} \geq R X \_T h r e s h$ are potential receivers of A, they will refrain from new communications (both send and receive) and wait for A's connection request. With the completions of ongoing receptions around A, $P t_{\text {bound }}$ of A gradually increases. When it becomes greater than $P t_{d e s}$, A is eligible to access the channel. On the other hand, because nodes sensing $P r_{B T t}<R X \_$Thresh will not be the receivers of $\mathrm{A}$, they can set up new connections. Their transmissions will not collide with the later transmission from A, since interference from node $\mathrm{A}$ has been considered.

\section{Simulation Results}

In our simulation, we study the performance of our protocol using NS2.27, and compare it with other protocols.

In the test environment, we use the default settings of wireless channel and physical layer in NS2.27. The antenna gain is 1 , the height of each antenna is $1.5 \mathrm{~m}$, the system loss is 1 and the carrier frequency is $916 \mathrm{MHz}$. The $R X_{-}$Thresh is $-64 \mathrm{dBm}$, CS_Thresh is $-78 \mathrm{dBm}$, SIR_Thresh is $10 \mathrm{~dB}$ and the maximal transmission power is $24.5 \mathrm{dBm}$. The propagation model is the two-ray-ground model. With these settings, the maximal transmission range is $250 \mathrm{~m}$.

We construct a network with an area of $1000 \mathrm{~m} \times 1000 \mathrm{~m}$, within which 100 nodes are randomly distributed, and 100 onehop flows are randomly generated to remove the influence of routing. All flows follow the Poisson traffic model with arrival rate $\lambda$. The bandwidth of the data channel is $1 \mathrm{Mbps}$ and the bandwidth of the busy tone channel is ignored as discussed in Section III. The length of a data packet is 1000 bytes. The maximal queue length for each node is 50 packets. The simulation time of a test is $60 \mathrm{~s}$, and we only use the data after $10 \mathrm{~s}$ to avoid the system transients.

We first present the throughput of our protocol working at the basic mode and compare it with other MAC protocols. In this section, throughput and offered traffic load are normalized by the channel bandwidth.

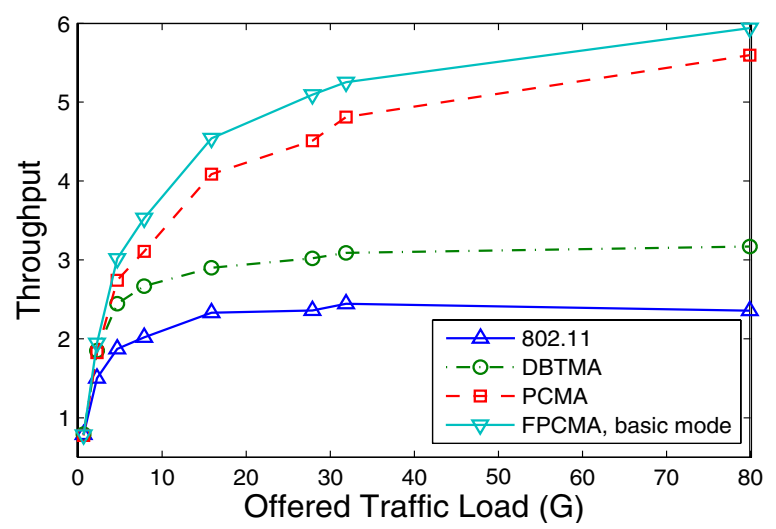

Fig. 1. Throughput comparison between 802.11, DBTMA, PCMA and the basic mode of FPCMA.

As shown in Fig. 1, 802.11 has the lowest throughput, since it suffers from both the hidden and exposed terminal problems. DBTMA has an improvement of about $30 \%$ with respect to 802.11 by solving the hidden and exposed terminal problems. With the help of power control, PCMA almost doubles the throughput of DBTMA. Throughput of our protocol is about $10 \%$ higher than PCMA since RTS packet is protected by $B T_{t}$ and there is no CTS blocking problem.

Now, we study the performance of the fair mode of our protocol. To measure fairness, we divide the flows into five groups according to their distances and use Jain's fairness index [10] as an evaluation metric.

$$
\text { fairness index }=\frac{\left(\sum_{i=1}^{n} T_{i}\right)^{2}}{n * \sum_{i=1}^{n}\left(T_{i}\right)^{2}}
$$

where $n$ is the total number of flows within a group and $T_{i}$ is the throughput of a flow. It is most fair when the fairness index is 1 , and less fair with lower index values.

From Fig. 2, we observe that DBTMA is a fair protocol and PCMA suffers severely from the unfairness problem. We also find that the unfairness problem is greatly alleviated by our FPCMA, especially with smaller F_Thresh. When $F \_$Thresh $=1$, our protocol has better fairness than DBTMA. 


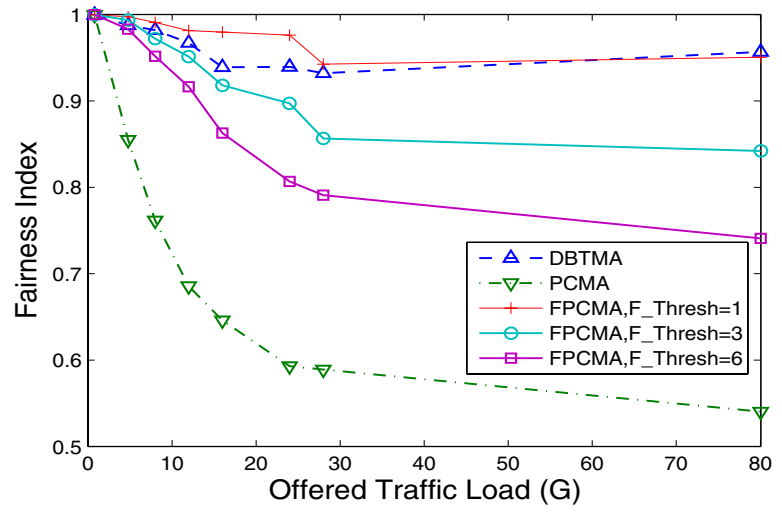

Fig. 2. Fairness comparison between DBTMA, PCMA and FPCMA with different F_Thresh.

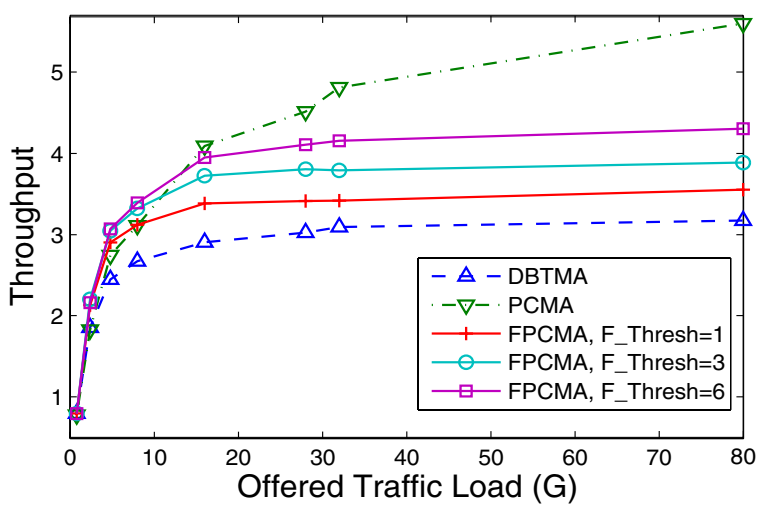

Fig. 3. Throughput comparison between DBTMA, PCMA and FPCMA with different F_Thresh.

We also find that fairness is achieved at the price of throughput: the better the fairness, the more throughput degradation. Comparing Fig. 1 with Fig. 3, we can see that the throughput of FPCMA changes from higher than PCMA to lower than PCMA, when the fairness mechanism is used. The earlier the fairness mode is used, the smaller the throughput gain. Thus the key of our protocol is to select a proper F_Thresh for switching from the basic mode to the fairness mode. When fairness is preferred, we should select a smaller F_Thresh, and vice versa.

\section{CONCLUSION}

In this paper, we propose a fair power controlled MAC protocol in MANET. There are two modes in our protocol: a basic mode and a fair mode. In the basic mode, dual busy tone is introduced to protect the RTS packet and solve the CTS blocking problem. The basic mode can provide better throughput than the conventional PCMA protocol. In the fair mode, we resolve the unfairness problem in PCMA by using the busy tone as a mechanism for channel access. To balance between throughput and fairness, nodes can choose to switch between the two modes based on the number of unsuccessful retries. This number can be used to trade off fairness and through- put. The smaller the number is, the better the fairness and less throughput, and vice versa.

\section{APPENDIX}

\section{A. Prooffor BT Reply Mechanism}

Theorem 1: When node B sets up $B T_{r}$ as a reply to RTS from node $A$, node $A$ will sense the maximum received power on $B T_{r}$ channel to be increased by a factor of at least SIR_Thresh.

Proof: Let $\max \left\{\operatorname{Pr}_{B T r}\right\}$ be the maximal received power on $B T_{r}$ channel before node A sends RTS, from which node A can deduce RTS transmission power $P t_{\text {bound }}$ from (3). According to the propagation model in (4), the RTS power received by node $\mathrm{B}$ is given by

$$
\operatorname{Pr}_{R T S}=C_{k} \cdot P t_{\text {bound }} \cdot d^{-k}
$$

Assuming there is no noise at node $\mathrm{B}$, i.e., $P_{n}=0, B T_{r}$ power of node B can be calculated according to (1) and (2) as

$$
P t_{B T r}^{\prime}=\frac{P t_{\max } \cdot C S_{-} \text {Thresh }}{\left(P r_{R T S} / S I R \_ \text {Thresh }\right)} .
$$

Note $P t_{B T r}^{\prime}$ is the minimum transmission power that will be used by node $\mathrm{B}$. With non-zero $P_{n}, P t_{B T r}^{\prime}$ will be increased accordingly. Then node A senses the $B T_{r}$ power from node B at

$$
\operatorname{Pr}_{B T r}^{\prime}=C_{k} \cdot P t_{B T r}^{\prime} \cdot d^{-k} .
$$

Combining above formulae together with (3), we get

$$
\operatorname{Pr}_{B T r}^{\prime}=S I R \_T h r e s h \cdot \max \left\{\operatorname{Pr}_{B T r}\right\}
$$

which means the received power of $\operatorname{Pr}_{B T r}^{\prime}$ is SIR_Thresh times greater than $\max \left\{P r_{B T r}\right\}$.

Obviously, when $P_{n} \neq 0$, this ratio will be even higher.

\section{REFERENCES}

[1] The editors of IEEE 802.11, ANSI/IEEE Std 802.11, 1999 Edn. Part 11: Wireless LAN Medium Access Control (MAC) and Physical Layer (PHY) specifications.

[2] X. Yang and N.H. Vaidya, "Explicit and Implicit Pipelining for Wireless Medium Access Control,' Proc. VTC'03 fall, Orlando, Florida, USA, Oct. 2003, pp. 1427-1431.

[3] S. Singh and C.S. Raghavendra, "PAMAS - Power Aware Multi-access Protocol with Signalling for Ad Hoc Networks," ACM Computer Communication Review, Vol. 28, Issue 3, pp. 5-26, Jul. 1998.

[4] C. Wu and V.O.K. Li, "Receiver-initiated Busy-tone Multiple Access in Packet Radio Networks," Proc. ACM SIGCOMM'87, Stowe, Vermont, USA, Aug. 1987, pp. 336-342.

[5] Z. Haas and J. Deng, "Dual Busy Tone Multiple Access (DBTMA): A Multiple Access Control Scheme for Ad Hoc Networks," IEEE Trans. Commun., Vol. 50, No. 6, pp. 975-984, Jun. 2002.

[6] J. Monks, V. Bharghavan and W.-M. Hwu, "A Power Controlled Multiple Access Protocol for Wireless Packet Networks," Proc. IEEE INFOCOM'01, Anchorage, Alaska, USA, Apr. 2001, pp. 219-228.

[7] S. L. Wu, Y. C. Tseng and J. P. Sheu, "Intelligent Medium Access for Mobile Ad Hoc Networks with Busy Tone and Power Control," IEEE J. Select. Areas Commun., Vol. 18, No. 9, pp. 1647-1656, Sept. 2000.

[8] F. A. Tobagi and L. Kleinrock, "Packet Switching in Radio Channels: Part II-The Hidden Terminal Problem in Carrier Sense Multiple-Access and the Busy-Tone Solution," IEEE Trans. Commun., Vol. COM-23, No. 12, pp. 1417-1433, Dec. 1975.

[9] J. Gomez, A. T. Campbell, M. Naghshineh and C. Bisdikian, "PARO: Supporting Dynamic Power Controlled Routing in Wireless Ad Hoc Networks," ACM/Kluwer Journal on Wireless Networks(WINET), Vol. 9, Issue 5, pp. 443-460, Sept. 2003.

[10] R. Jains, The Art of Computer Systems Performance Analysis, John Wiley \& Sons, 1991 